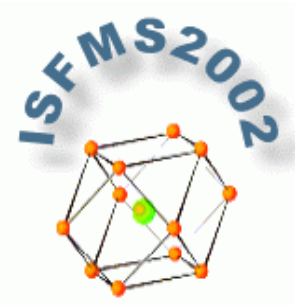

\title{
molecules
}

ISSN 1420-3049

http://www.mdpi.org/

\section{Valence Topological Charge-Transfer Indices for Dipole Moments}

\section{Francisco Torrens}

Institut Universitari de Ciència Molecular, Universitat de València, Dr. Moliner 50, E-46100 Burjassot (València), Spain. http://www.uv.es/ icmol. Tel. +34 963543 182, Fax +34 963543 156, E-mail Francisco.Torrens@uv.es

\begin{abstract}
New valence topological charge-transfer indices are applied to the calculation of dipole moments. The algebraic and vector semisum charge-transfer indices are defined. The combination of the charge-transfer indices allows the estimation of the dipole moments. The model is generalized for molecules with heteroatoms. The ability of the indices for the description of the molecular charge distribution is established by comparing them with the dipole moments of a homologous series of phenyl alcohols. Linear and non-linear correlation models are obtained. The new charge-transfer indices improve the multivariable non-linear regression equations for the dipole moment. When comparing with previous results, the variance decreases 92\%. No superposition of the corresponding $G_{k}-J_{k}$ and $G_{k}^{V}-J_{k}^{V}$ pairs is observed. This diminishes the risk of co-linearity. Inclusion of the oxygen atom in the $\pi$-electron system is beneficial for the description of the dipole moment, owing to either the role of the additional $p$ orbitals provided by the heteroatom or the role of steric factors in the $\pi$-electron conjugation. Linear and non-linear correlations between the fractal dimension and various descriptors point not only to a homogeneous molecular structure but also to the ability to predict and tailor drug properties.
\end{abstract}

Keywords: Dipole moment, molecular charge distribution, valence topological chargetransfer index, transdermal drug delivery, phenyl alcohol. 


\section{Introduction}

The rationale for transdermal drug delivery needs to be carefully identified [1]. There has been great interest in developing systems for controlled delivery of drugs and other bioactive substances [2]. One technique is to attach a specialized patch on the skin with uncoated polymer matrices containing the embedded drug [3]. Other technique involves covering most of the matrix with an impermeable material [4]. The pathways that exist in a porous membrane used to deliver drugs over a continuous period form a percolating path with usual fractal structures $[5,6]$.

Percutaneous penetration depends essentially on the lipophilicity of the substances tested [7]. The use of homologous series of compounds made it possible to establish behaviour models that allow predicting the percutaneous absorption capacity of chemically related substances [8,9]. Much of the theoretical background information on percutaneous absorption was developed from studies of nonelectrolytic permeating species [10,11]. However, the vehicle's $\mathrm{pH}$ may have a profound influence upon the percutaneous delivery from topical products [12]. Depending on the $\mathrm{pK}_{\mathrm{a}}$ of the compound and on the $\mathrm{pH}$ of the vehicle, an equilibrium mixture of ionized and unionized species would be present in the immediate vicinity of the skin [13]. The permeation of ionized drugs through the skin must be taken into account [14]. Penetration enhancers may act by either interacting with the highly ordered lipid structure or modifying the partitioning of the drug into the tissue $[15,16]$.

Different phenyl alcohols are included in many pharmaceutical and cosmetic products, e.g., bacteriostatic agents and essential oils. Benzyl alcohol, which is used as a bacteriostatic agent and also as a solvent, was evaluated as a percutaneous enhancer [17]. López et al. assessed other phenyl alcohols as enhancers for 5-fluorouracil [8], which was used in in vitro diffusion experiments [18]. Other authors employed dipalmitoylphosphatidylcholine as enhancer for azone [19].

In previous papers, molecular topological charge-transfer indices were applied to the calculation of the molecular dipole moment of hydrocarbons [20]. New valence charge-transfer indices were introduced to take into account the presence of heteroatoms in the molecules and applied to valenceisoelectronic series of benzene and styrene [21]. In this paper, the method has been applied to the calculation of the dipole moment of a homologous series of phenyl alcohols. In the next section, the topological charge-transfer indices are revised. Next, the valence charge-transfer indices for heteroatoms are presented. Following that, the calculation results are discussed. The last section summarizes my conclusions.

\section{Topological charge-transfer indices}

The two most important matrices that delineate the labelled chemical graph are the adjacency $(\overline{\bar{A}})$ [22-24] and the distance $(\overline{\bar{D}})$ matrices, wherein $D_{i j}=I_{i j}$ if $i=j$, " 0 " otherwise; $l_{i j}$ is the shortest edge count between vertices $i$ and $j$ [25]. In $\overline{\bar{A}}, A_{i j}=1$ if vertices $i$ and $j$ are adjacent, " 0 " otherwise. The $\overline{\bar{D}}^{*}$ matrix is the matrix whose elements are the squares of the reciprocal distances $D_{i j}^{-2}$ [26]. Now, the intermediate matrix $\overline{\bar{M}}$ is defined as the matrix product of $\overline{\bar{A}}$ by $\overline{\bar{D}}^{*}$ : 
$\overline{\bar{M}}=\overline{\bar{A}} \times \overline{\bar{D}} *$

The charge-transfer matrix $\overline{\bar{C}}$ is defined as $\overline{\bar{C}}=\overline{\bar{M}}-\overline{\bar{M}}^{T}$, where $\overline{\bar{M}}^{T}$ is the transpose of $\overline{\bar{M}}$ [27]. By agreement, $C_{i i}=M_{i i}$. For $i$ ? $j$, the $C_{i j}$ terms represent a measure of the intramolecular net charge transferred from atom $j$ to $i$.

Gálvez et al. defined the topological charge-transfer indices $G_{k}$ as the sum, in absolute value, of the $C_{i j}$ terms defined for the vertices $i, j$ placed at a topological distance $D_{i j}$ equal to $k$ :

$G_{k}=\sum_{i=1}^{N-1} \sum_{j=i+1}^{N}\left|C_{i j}\right| \delta\left(k, D_{i j}\right)$

where $N$ is the number of vertices in the graph, $D_{i j}$ are the entries of the $\overline{\bar{D}}$ matrix, and $\delta$ is the Kronecker $\delta$, being $d=1$ for $i=j$ and $d=0$ for $i ? j . G_{k}$ represents the sum of all the $C_{i j}$ terms, for every pair of vertices $i$ and $j$ at topological distance $k$. Gálvez et al. also introduced other topological charge-transfer index, $J_{k}$, as

$J_{k}=\frac{G_{k}}{N-1}$

This index represents the mean value of the charge transfer for each edge, since the number of edges for acyclic compounds is $N-1$. The $G_{k}$ and $J_{k}$ indices were applied to the calculation of the molecular dipole moment of hydrocarbons, the boiling temperature of hydrocarbons and alcohols, the vaporization enthalpy of alkanes, the activity of drugs [28-30], the capacity and separation factors in chromatographic chiral separations of biomolecules [31], the resonance energy of hydrocarbons, the $\log \mathrm{IC}_{50}$ of the $D_{2}$ dopamine receptor and the $\sigma$ receptor of piperidines and the sedative character of chiral barbiturates [32].

The algebraic semisum charge-transfer index $\mu_{\mathrm{alg}}$ is defined as $\mu_{\mathrm{alg}}=\frac{1}{2} \sum_{i=1}^{N-1} \sum_{j=i+1}^{N} A_{i j} C_{i j}=\frac{1}{2} \sum_{e=1}^{m} C^{e}$

where $C^{e}$ is the $C_{i j}$ index for vertices $i$ and $j$ connected by edge $e$. In the first equation, the sum extends for all pairs of adjacent vertices in the molecular graph. In the second expression, the sum extends for the $m$ edges in the molecular graph. Therefore, $\mu_{\mathrm{alg}}$ is intended as a measure of the molecular dipole moment calculated by algebraic semisum of $C^{e}$.

An edge-to-edge analysis of $\mu$ suggests that each edge dipole moment $\mu^{e}$ connecting vertices $i$ and $j$ can be evaluated from the corresponding edge $C^{e}$ index as

$\mu^{e}=\frac{1}{2} C^{e}$

Each edge dipole can be associated with a vector $\bar{\mu}^{e}$ in space. This vector has magnitude $\mu^{e}$, lies in the edge $e$ connecting vertices $i$ and $j$, and its direction is from $j$ to $i$. The molecular dipole moment vector $\bar{\mu}$ results the vector sum of the edge dipole moments as $\bar{\mu}=\sum_{e=1}^{m} \bar{\mu}^{e}=\frac{1}{2} \sum_{e=1}^{m} \bar{C}^{e}$

summed for all the $m$ edges in the molecular graph. The vector semisum charge-transfer index $\mu_{\mathrm{vec}}$ is defined as the norm of $\bar{\mu}$ : 
$\mu_{\mathrm{vec}}=N(\bar{\mu})=\left(\mu_{x}^{2}+\mu_{y}^{2}+\mu_{z}^{2}\right)^{1 / 2}$

Therefore, $\mu_{\mathrm{vec}}$ is intended as a measure of the molecular dipole moment calculated by vector semisum of edge $\bar{C}^{e}$.

As $\mu_{\text {vec }}$ is associated with a vector in space, the three-dimensional structure of the molecule is needed. The method followed to get it is outlined as follows.

1. Read the Cartesian coordinates of the atoms.

2. Determine which atoms are bonded to which other atoms. The distance between two atoms is calculated and, if it is less than a certain value, a bond is assumed to exist between the two atoms.

3. Build the charge transfer terms $C_{i j}$.

4. Calculate the vector semisum dipole moment $\mu_{\text {vec. }}$.

Therefore, $\mu_{\mathrm{vec}}$ is not a pure topological index. It is rather a linear combination of topological indices, but the coefficients are geometric descriptors.

\section{Valence charge-transfer indices for heteroatoms}

In valence charge-transfer indices terms, the presence of each heteroatom is taken into account by introducing its electronegativity value in the corresponding entry of the main diagonal of the adjacency matrix $\overline{\bar{A}}$. For each heteroatom $X$, its entry $A_{i i}$ is redefined as

$A_{i i}^{V}=2.2\left(\chi_{X}-\chi_{C}\right)$

to give a modified $\overline{\bar{A}}^{V}$ matrix, where $\chi_{X}$ and $\chi_{C}$ are the electronegativities of heteroatom $X$ and carbon, respectively, in Pauling units. Notice that the subtractive term keeps $A_{\mathrm{ii}}^{\mathrm{V}}=0$ for the carbon atom (Equation 5). Moreover, the multiplicative factor reproduces $A_{i i}^{V}=2.2$ for oxygen, which was taken as standard in previous works. From the modified $\overline{\bar{A}}^{V}$ matrix, the valence $\overline{\bar{M}}^{V}$ and $\overline{\bar{C}}^{V}$ matrices, $\mu_{a \mathrm{lg}}^{V}, \mu_{\mathrm{vec}}^{V}$ and topological charge-transfer indices $G_{k}^{V}$ and $J_{k}^{V}$ can be calculated by following the former procedure with the $\overline{\bar{A}}^{V}$ matrix. The $C_{i j}^{V}, G_{k}^{V}$ and $J_{k}^{V}$ descriptors are graph invariants. The main difference between $\mu_{\mathrm{vec}}$ and $\mu_{\mathrm{vec}}^{V}$ is that $\mu_{\mathrm{vec}}$ is sensitive only to the steric effect of the heteroatoms while $\mu_{\text {vec }}^{V}$ is sensitive to both electronic and steric effects.

\section{Calculation results and discussion}

The molecular charge-transfer indices $G_{k}, J_{k}, G_{k}^{V}$ and $J_{k}^{V}$ (with $k<6$ ) for a series of 13 phenyl alcohols ( 8 form a homologous series and 5 are congeneric) are reported in Table 1 . In the homologous series, $G_{1}$ and $G_{2}$ are sensitive to the presence of the alkyl chain. $G_{3}, G_{4}$ and $G_{5}$ indicate the presence of at least 2, 3 and 4 carbon atoms in the alkyl chain, respectively. $G_{1}^{V}$ is influenced by the presence of the alkyl chain. $G_{2}^{V}-G_{5}^{V}$ point out the presence of at least 2-5 carbon atoms in the alkyl chain. 
Table 1. Values of the $G_{k}$ and $J_{k}$ charge-transfer indices up to the fifth order for a homologous series of phenyl alcohols.

\begin{tabular}{lrlllll}
\hline Molecule & $\mathbf{N}$ & $\mathbf{G}_{\mathbf{1}}$ & $\mathbf{G}_{\mathbf{2}}$ & $\mathbf{G}_{\mathbf{3}}$ & $\mathbf{G}_{\mathbf{4}}$ & $\mathbf{G}_{\mathbf{5}}$ \\
\hline Phenol & 7 & 2.0000 & 0.8889 & 0.3750 & 0.2222 & 0.0000 \\
benzyl alcohol & 8 & 1.2500 & 6.7778 & 0.8125 & 0.4133 & 0.1250 \\
2-phenyl-1-ethanol & 9 & 1.2500 & 6.7778 & 0.8750 & 0.5644 & 0.2431 \\
3-phenyl-1-propanol & 10 & 1.2500 & 6.7778 & 0.8750 & 0.6044 & 0.3333 \\
4-phenyl-1-butanol & 11 & 1.2500 & 6.7778 & 0.8750 & 0.6044 & 0.3611 \\
5-phenyl-1-pentanol & 12 & 1.2500 & 6.7778 & 0.8750 & 0.6044 & 0.3611 \\
6-phenyl-1-hexanol & 13 & 1.2500 & 6.7778 & 0.8750 & 0.6044 & 0.3611 \\
7-phenyl-1-heptanol & 14 & 1.2500 & 6.7778 & 0.8750 & 0.6044 & 0.3611 \\
1-phenyl-2-propanol & 10 & 2.2500 & 6.7778 & 0.9375 & 0.7156 & 0.3611 \\
2-phenyl-2-propanol & 10 & 2.7500 & 7.2222 & 1.5625 & 0.7956 & 0.3750 \\
3-phenyl-2-propen-1-ol & 10 & 1.2500 & 8.2222 & 0.8750 & 0.4844 & 0.2708 \\
1-phenyl-1-pentanol & 12 & 1.7500 & 7.1111 & 1.3125 & 0.8756 & 0.4861 \\
1-phenyl-2-pentanol & 12 & 2.2500 & 7.0000 & 1.0625 & 0.7556 & 0.4792 \\
\hline
\end{tabular}

\begin{tabular}{llllll}
\hline Molecule & $\mathbf{J}_{\mathbf{1}}$ & $\mathbf{J}_{\mathbf{2}}$ & $\mathbf{J}_{\mathbf{3}}$ & $\mathbf{J}_{\mathbf{4}}$ & $\mathbf{J}_{\mathbf{5}}$ \\
\hline phenol & 0.3333 & 0.1481 & 0.0625 & 0.0370 & 0.0000 \\
benzyl alcohol & 0.1786 & 0.9683 & 0.1161 & 0.0590 & 0.0179 \\
2-phenyl-1-ethanol & 0.1563 & 0.8472 & 0.1094 & 0.0706 & 0.0304 \\
3-phenyl-1-propanol & 0.1389 & 0.7531 & 0.0972 & 0.0672 & 0.0370 \\
4-phenyl-1-butanol & 0.1250 & 0.6778 & 0.0875 & 0.0604 & 0.0361 \\
5-phenyl-1-pentanol & 0.1136 & 0.6162 & 0.0795 & 0.0549 & 0.0328 \\
6-phenyl-1-hexanol & 0.1042 & 0.5648 & 0.0729 & 0.0504 & 0.0301 \\
7-phenyl-1-heptanol & 0.0962 & 0.5214 & 0.0673 & 0.0465 & 0.0278 \\
1-phenyl-2-propanol & 0.2500 & 0.7531 & 0.1042 & 0.0795 & 0.0401 \\
2-phenyl-2-propanol & 0.3056 & 0.8025 & 0.1736 & 0.0884 & 0.0417 \\
3-phenyl-2-propen-1-ol & 0.1389 & 0.9136 & 0.0972 & 0.0538 & 0.0301 \\
1-phenyl-1-pentanol & 0.1591 & 0.6465 & 0.1193 & 0.0796 & 0.0442 \\
1-phenyl-2-pentanol & 0.2045 & 0.6364 & 0.0966 & 0.0687 & 0.0436 \\
\hline & & & & & \\
\hline Molecule & $G_{1}^{V}$ & $G_{2}^{V}$ & $G_{3}^{V}$ & $G_{4}^{V}$ & $G_{5}^{V}$ \\
\hline phenol & 2.2000 & 1.1000 & 0.3639 & 0.0847 & 0.0000 \\
benzyl alcohol & 2.9500 & 6.6611 & 0.5625 & 0.2533 & 0.0370 \\
2-phenyl-1-ethanol & 2.9500 & 7.1056 & 0.7444 & 0.4044 & 0.1319 \\
3-phenyl-1-propanol & 2.9500 & 7.1056 & 0.9944 & 0.5019 & 0.2222 \\
\hline
\end{tabular}




\begin{tabular}{llllll}
\hline 4-phenyl-1-butanol & 2.9500 & 7.1056 & 0.9944 & 0.6619 & 0.2824 \\
5-phenyl-1-pentanol & 2.9500 & 7.1056 & 0.9944 & 0.6619 & 0.3936 \\
6-phenyl-1-hexanol & 2.9500 & 7.1056 & 0.9944 & 0.6619 & 0.3936 \\
7-phenyl-1-heptanol & 2.9500 & 7.1056 & 0.9944 & 0.6619 & 0.3936 \\
1-phenyl-2-propanol & 3.4500 & 7.6556 & 0.8069 & 0.5556 & 0.2500 \\
2-phenyl-2-propanol & 3.4500 & 8.2056 & 1.3125 & 0.6356 & 0.2870 \\
3-phenyl-2-propen-1-ol & 2.9500 & 8.3278 & 0.6306 & 0.3819 & 0.1597 \\
1-phenyl-1-pentanol & 2.9500 & 7.3222 & 1.1819 & 0.7731 & 0.4861 \\
1-phenyl-2-pentanol & 3.4500 & 7.6556 & 1.0514 & 0.7331 & 0.3681 \\
\hline & & & & & \\
\hline Molecule & $J_{1}^{V}$ & $J_{2}^{V}$ & $J_{3}^{V}$ & $J_{4}^{V}$ & $J_{5}^{V}$ \\
\hline phenol & 0.3637 & 0.1833 & 0.0606 & 0.0141 & 0.0000 \\
benzyl alcohol & 0.4214 & 0.9516 & 0.0804 & 0.0362 & 0.0053 \\
2-phenyl-1-ethanol & 0.3688 & 0.8882 & 0.0931 & 0.0506 & 0.0165 \\
3-phenyl-1-propanol & 0.3278 & 0.7895 & 0.1105 & 0.0558 & 0.0247 \\
4-phenyl-1-butanol & 0.2950 & 0.7106 & 0.0994 & 0.0662 & 0.0282 \\
5-phenyl-1-pentanol & 0.2682 & 0.6460 & 0.0904 & 0.0602 & 0.0358 \\
6-phenyl-1-hexanol & 0.2458 & 0.5921 & 0.0829 & 0.0552 & 0.0328 \\
7-phenyl-1-heptanol & 0.2269 & 0.5466 & 0.0765 & 0.0509 & 0.0303 \\
1-phenyl-2-propanol & 0.3833 & 0.8506 & 0.0897 & 0.0617 & 0.0278 \\
2-phenyl-2-propanol & 0.3833 & 0.9117 & 0.1458 & 0.0706 & 0.0319 \\
3-phenyl-2-propen-1-ol & 0.3278 & 0.9253 & 0.0701 & 0.0424 & 0.0177 \\
1-phenyl-1-pentanol & 0.2682 & 0.6657 & 0.1074 & 0.0703 & 0.0442 \\
1-phenyl-2-pentanol & 0.3136 & 0.6960 & 0.0956 & 0.0666 & 0.0335 \\
\hline
\end{tabular}

The molecular dipole moments $\mu$ (experimental and calculated as vector semisums of $C_{i j}$ or $C_{i j}^{V}$ ) are listed in Table 2 As experimental values are not available for all the series, some reference values are calculated with program MOPAC-AM1. The reliability of the results has been tested with the first four entries in Table 2, for which experimental data are available. AM1 calculations adequately reproduce the oscillatory behaviour of the experimental data, mimicking two minima for phenol and 2-phenyl-1-ethanol and two maxima for benzyl alcohol and 3-phenyl-1-propanol. This test suggests that AM1 allows a good approximation, at least for the general performance of the homologous series as a whole, and that the error is sufficiently constant throughout the homologous series. The number of compounds in the homologous series has not been increased because longer phenyl alcohols are not percutaneous enhancers owing to their lower transdermal penetration. In particular, 3-phenyl-2-propen-1-ol has been chosen in order to compare the influence of a double bound in the alkyl chain region of a phenyl alcohol. The experimental $\mu$ decreases $c a$. 3\% from that of 3-phenyl-1-propanol due to the presence of the double bond. The presence of an enol group (conjugated double bond in $\beta$ position with respect to the -OH group) lends 3-phenyl-2-propen-1-ol to 
particular structural characteristics (greater rigidity than for 3-phenyl-1-propanol) with a lower $\mu$. For

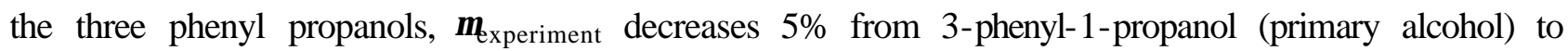
1-phenyl-2-propanol (secondary alcohol) and $11 \%$ to 2-phenyl2-propanol (tertiary alcohol). For the

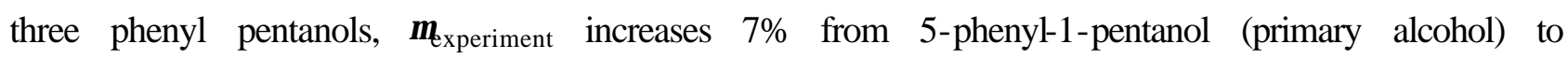
1-phenyl-1-pentanol (secondary alcohol with both -phenyl and - $\mathrm{OH}$ groups in terminal position) and decreases $8 \%$ to 1-phenyl 2-pentanol (secondary alcohol).

Table 2. Molecular dipole moment values, $\mu$ (D), for a series of phenyl alcohols with charge-transfer indices.

\begin{tabular}{lllll}
\hline Molecule & $\begin{array}{l}\text { Number of carbon } \\
\text { atoms in alkyl chain }\end{array}$ & $\begin{array}{l}\text { Vector } \\
\text { semisum }\end{array}$ & $\begin{array}{l}\text { Valence vector } \\
\text { semisum }\end{array}$ & Experiment $^{\mathrm{a}}$ \\
\hline phenol & 0 & 0.737 & 2.431 & $1.400\left(1.233^{\mathrm{b}}\right)$ \\
benzyl alcohol & 1 & 0.589 & 2.487 & $1.700\left(1.568^{\mathrm{b}}\right)$ \\
2-phenyl-1-ethanol & 2 & 0.700 & 2.257 & $1.590\left(1.497^{\mathrm{b}}\right)$ \\
3-phenyl-1-propanol & 3 & 0.573 & 2.519 & $1.640\left(1.597^{\mathrm{b}}\right)$ \\
4-phenyl-1-butanol & 4 & 0.702 & 2.249 & $1.345^{\mathrm{b}}$ \\
5-phenyl-1-pentanol & 5 & 0.573 & 2.519 & $1.626^{\mathrm{b}}$ \\
6-phenyl-1-hexanol & 6 & 0.702 & 2.250 & $1.346^{\mathrm{b}}$ \\
7-phenyl-1-heptanol & 7 & 0.573 & 2.518 & $1.634^{\mathrm{b}}$ \\
1-phenyl-2-propanol & 3 & 0.923 & 2.347 & $1.564^{\mathrm{b}}$ \\
2-phenyl-2-propanol & 3 & 0.780 & 2.821 & $1.463^{\mathrm{b}}$ \\
3-phenyl-2-propen-1-ol & 3 & 0.561 & 2.495 & $1.591^{\mathrm{b}}$ \\
1-phenyl-1-pentanol & 5 & 0.426 & 2.794 & $1.746^{\mathrm{b}}$ \\
1-phenyl-2-pentanol & 5 & 0.895 & 2.367 & $1.496^{\mathrm{b}}$ \\
\hline
\end{tabular}

${ }^{\mathrm{a}}$ Taken from Reference 60.

${ }^{\mathrm{b}}$ Calculations carried out with program MOPAC-AM1.

Figure 1 illustrates how the dipole moment of the homologous phenyl alcohols fluctuates with the number of $\mathrm{C}$ atoms in the alkyl chain, $n$. Experimental $\mu$ and calculated $\mu_{\mathrm{vec}}^{V}$ vary in an alternate

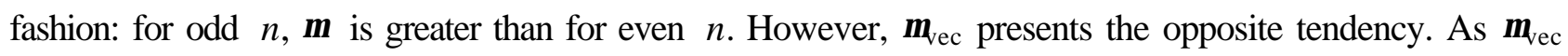
is not sensitive to the electronic effect of the oxygen atom, it is clear that the steric $\left(\mu_{\mathrm{vec}}\right)$ and electronic $\left(\mu_{\mathrm{vec}}^{V}\right)$ factors are antagonistic, and that the electronic effect dominates over the steric one. The

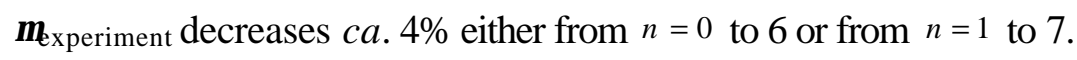


Figure 1. Dipole moment of homologous phenyl alcohols as a function of the number of $\mathrm{C}$ atoms in alkyl chain.

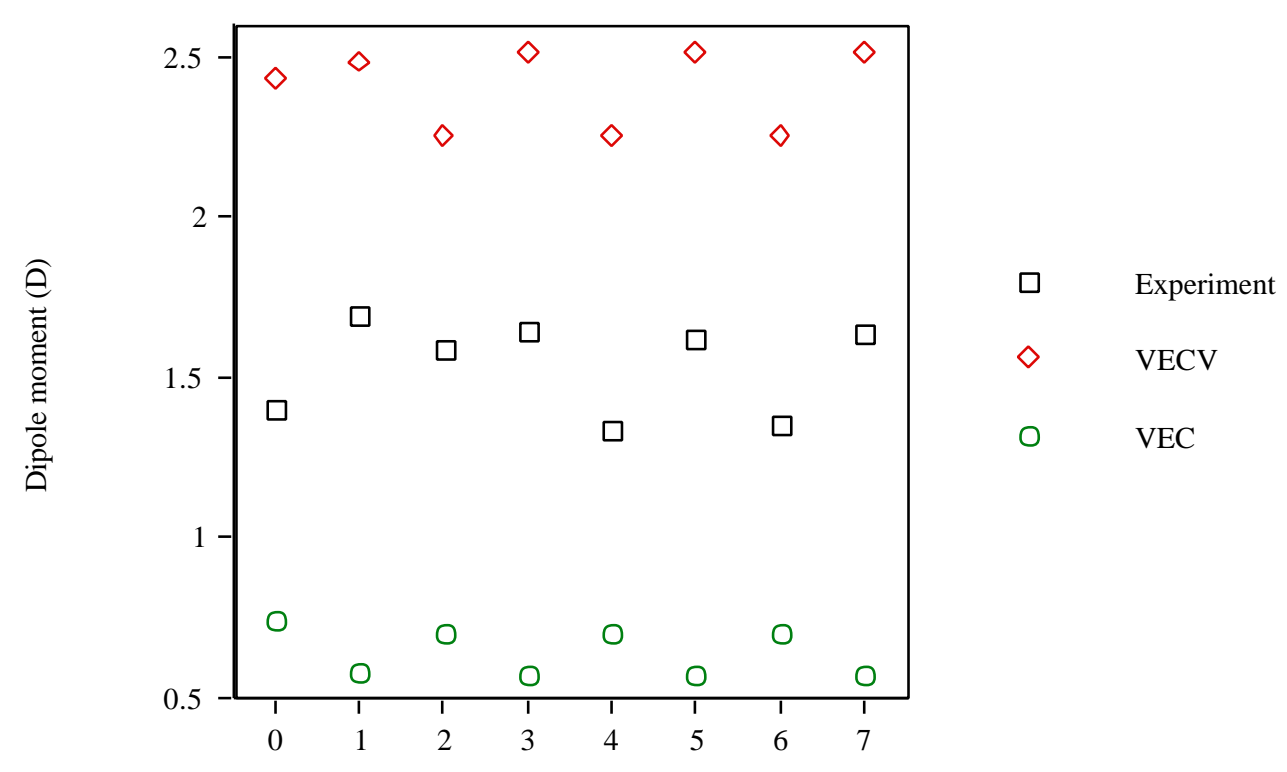

Number of $\mathrm{C}$ atoms in alkyl chain

The experimental dipole moments and charge-transfer indices have been correlated for the homologous series. The best linear regression results:

$\mu=1.08+1.32 G_{3}-12.5 J_{4}^{V}$

$n=8$

$r=0.701$

$\mathrm{SD}=0.123$

$F=2.4$

The mean absolute percentage error (MAPE) is $4.53 \%$ and the approximation error variance (AEV) is 0.5087. All other models with greater MAPE and AEV have been discarded. The presence of chargetransfer $\left(G_{3}\right)$ and valence $\left(J_{4}^{V}\right)$ indices is representative of charge-transfer processes and clearly conditions the polar character of the correlated compounds. The best non-linear model for $\mu$ does not improve the results.

If the congeneric set is included in the model of phenyl alcohols, the best linear regression for all $\mu$ results:

$\mu=1.19+0.0503 G_{2}$

MAPE $=5.52 \%$

$\mathrm{AEV}=0.6142$

$n=13$

$r=0.621$

$\mathrm{SD}=0.115$

$F=6.9$

and AEV increases $21 \%$. The best non-linear model for $\mu$ does not improve the results.

If all $\mu$ are calculated with AM1, the best linear regression results: $\mu=1.19+0.0481 G_{2}$

MAPE $=6.09 \%$

$\mathrm{AEV}=0.5721$

$n=8$

$r=0.654$

$\mathrm{SD}=0.125$ $F=4.5$

and AEV augments $12 \%$. The best non-linear model for $\mu$ does not improve the results.

If $\mu_{\mathrm{vec}}$ is included in the model of phenyl alcohols, the best linear regression for $\mu$ results: 


$$
\begin{array}{lllr}
\mu=2.41+0.895 J_{1}^{V}-1.80 \mu_{\mathrm{vec}} & \text { MAPE }=2.66 \% & \text { AEV }=0.1430 \\
n=8 & r=0.926 & \mathrm{SD}=0.065 & F=15.0
\end{array}
$$

and AEV decreases $72 \%$. Notice that the negative sign of the $\mu_{\mathrm{vec}}$ coefficient is due to the opposite

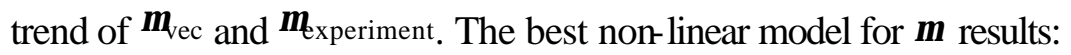
$\mu=1.54+0.144 z_{12}-0.0502 z_{11} z_{12}$

$$
\begin{aligned}
& z_{11}=-5.41+97.0 J_{4} \\
& z_{12}=9.55-13.8 \mu_{\mathrm{vec}}-2.48\left(G_{4}^{V}\right)^{2}
\end{aligned}
$$

$$
\text { MAPE }=1.52 \%
$$

$$
\mathrm{AEV}=0.0715
$$

and AEV diminishes $86 \%$.

If $\mu_{\mathrm{vec}}^{V}$ is included in the model, the best linear regression for $\mu$ results:

$$
\begin{array}{lllr}
\mu=-0.799+0.364 J_{2}^{V}+0.871 \mu_{\text {vec }}^{V} & \text { MAPE }=2.59 \% & \text { AEV }=0.1351 \\
n=8 & r=0.930 & \mathrm{SD}=0.064 & F=16.0
\end{array}
$$

$n=8 \quad r=0.930 \quad \mathrm{SD}=0.06$
and AEV drops $73 \%$. The non-linear model with $\mu_{\text {vec }}^{V}$ results:

$\mu=1.54+0.140 z_{12}+0.0143 z_{11} z_{12}$

$$
\begin{aligned}
& z_{11}=-4.72+54.5 J_{3} \\
& z_{12}=-50.4+51.7 J_{2}+20.1 \mu_{\text {vec }}^{\mathrm{v}}-20.2 J_{2} \mu_{\text {vec }}^{\mathrm{v}}
\end{aligned}
$$$$
\text { MAPE }=1.38 \%
$$$$
\mathrm{AEV}=0.0410
$$

and AEV decreases 92\%. Due to the complexity of Equations 8 and 10, a Fortran program has been written to calculate $\mu$. The program is available from the author at Internet (Francisco.Torrens@uv.es).

No superposition of the corresponding $G_{k}-J_{k}$ and $G_{k}^{V}-J_{k}^{V}$ pairs is observed in Equations 6-10. This diminishes the risk of co-linearity in the fit, given the close relationship between each pair $G_{k}, J_{k}$ in Equation 2 [33].

The correlation coefficient found between cross-validated representatives and the property values $R_{\mathrm{cv}}$ has been calculated with the leave- $n$-out procedure for Equations 6-10 [34]. The procedure furnishes a new method for selecting the best set of descriptors according to the criterion of maximization of the value of $R_{\mathrm{cv}}$. The $R_{\mathrm{cv}}$ calculations for a homologous series of phenyl alcohols are given in Table 3 for $1 \leq n \leq 5$. In general, $R_{\mathrm{cv}}$ decreases with $n$. In particular, Equations 9-10 give greater $R_{\mathrm{cv}}$ than Equations $7-8(2 \%)$ and than Equation $6(86 \%)$ for the whole range of $n$ given in Table 3, although the results for Equations 7 and 9 are rather similar. The corresponding interpretation is that Equations 9-10 are more predictive than Equations 7-8 or 6.

Table 3. Cross-validation correlation coefficient in a leave- $n$-out procedure for a homologous series of phenyl alcohols.

\begin{tabular}{lllllc}
\hline $\mathbf{n}$ & Equation 6 & Equation 7 & Equation 8 & Equation 9 & Equation 10 \\
\hline 1 & 0.477 & 0.834 & 0.915 & 0.823 & 0.956 \\
2 & - & 0.827 & 0.916 & 0.821 & 0.954 \\
3 & - & 0.819 & 0.916 & 0.821 & 0.951 \\
4 & - & 0.806 & 0.915 & 0.820 & 0.935 \\
5 & - & - & 0.890 & 0.823 & -0.951 \\
\hline
\end{tabular}


In the rest of this section, new parameters are calculated to illustrate the importance of linear and non-linear models in pharmacology. Another intramolecular property related with charge-transfer is the quadrupole moment tensor $\Theta$, defined by

$\Theta_{a b}=\Theta_{b a}=\frac{1}{2} \sum_{i} q^{i}\left[3 r_{a}^{i} r_{b}^{i}-\left(r^{i}\right)^{2} \delta_{a b}\right]$

where $q^{i}$ is the $i$-th element of charge at the point $\vec{r}^{i}$ relative to an origin fixed at the centre of mass in the molecule [35]. The $\delta_{a b}$ stands for the Kronecker $\delta$. The subscripts $a, b \ldots$ denote vector or tensor components and can be equal to the Cartesian components $x, y, z$. Tensor $\Theta$ is calculated with our program POLAR [36,37]. The fractal dimension $D$ of the solvent-accessible surface $A_{s}$ of the phenyl alcohols may then be obtained according to Lewis and Rees as

$D=2-\frac{d\left(\log \mathrm{A}_{\mathrm{s}}\right)}{d(\log R)}$

where $R$ is the radius of a probe representing the solvent [38]. It is calculated with our program TOPO. The fractal dimension $D$ provides a quantitative indication of the degree of surface accessibility toward different solvents [39-46]. In previous papers, the fractal dimension of transdermal-delivery drug models (phenyl alcohols [47] and 4-alkylanilines [48]) was calculated. A linear model for the molecular quadrupole moment of the homologous phenyl alcohols vs. fractal dimension is shown in Figure 2.

Figure 2. Quadrupole moment of homologous phenyl alcohols $v s$. the fractal dimension.

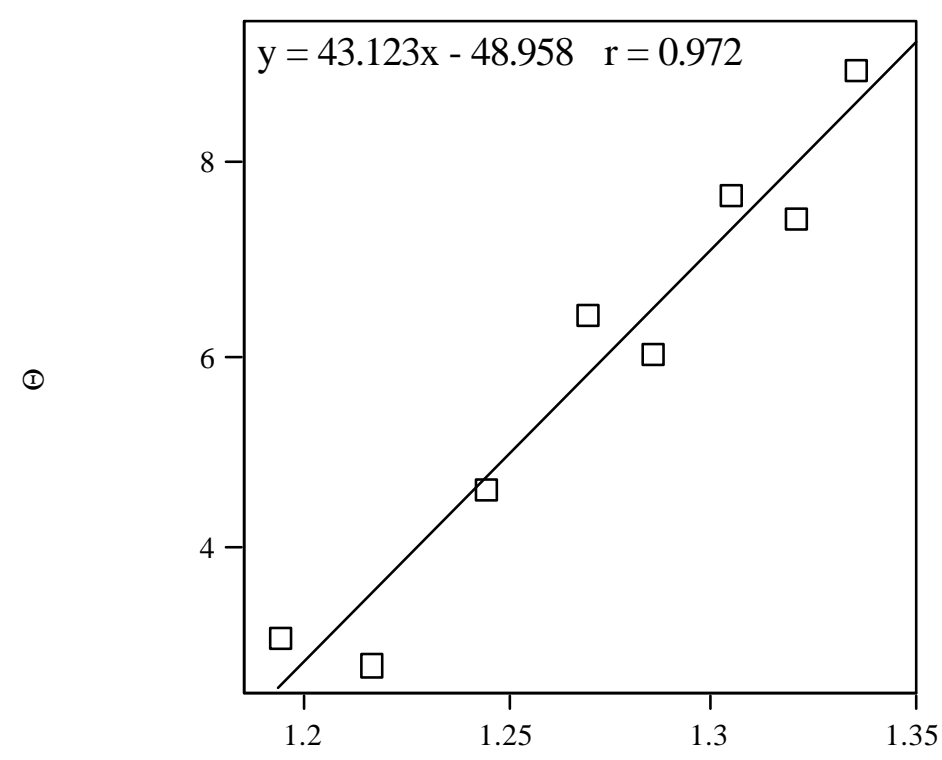

$\mathrm{D}$

The 1-octanol-water partition coefficient of a substance soluble in 1-octanol and water is defined as $P=\frac{a_{\text {l-octanol }}}{a_{\text {water }}}$ 
where $a_{1-\text { octanol }}$ and $a_{\text {water }}$ are the activities of the solute in 1-octanol and water, respectively [49]. $P$ is calculated with the equation:

$R T \ln P=\Delta G_{\text {solv }}^{o}$ (water) $-\Delta G_{\text {solv }}^{o}(1$ - octanol)

at a given $T$, which is taken as $298 \mathrm{~K} . R$ is the gas constant, and $\Delta G_{\text {solv }}^{o}\left(1-\right.$ octanol) and $\Delta G_{\text {solv }}^{o}$ (water) (in $\mathrm{kJ} \cdot \mathrm{mol}^{-1}$ ) are the standard-state free energies of solvation of a given solute considered in 1-octanol and water, respectively. The solvation energies are calculated with our program SCAP [50-55]. A non-linear model for $\log P$ of the homologous phenyl alcohols $v s$. fractal dimension is illustrated in Figure 3.

Figure 3. 1-Octanol-water partition coefficient of homologous phenyl alcohols $v s$. the fractal dimension.

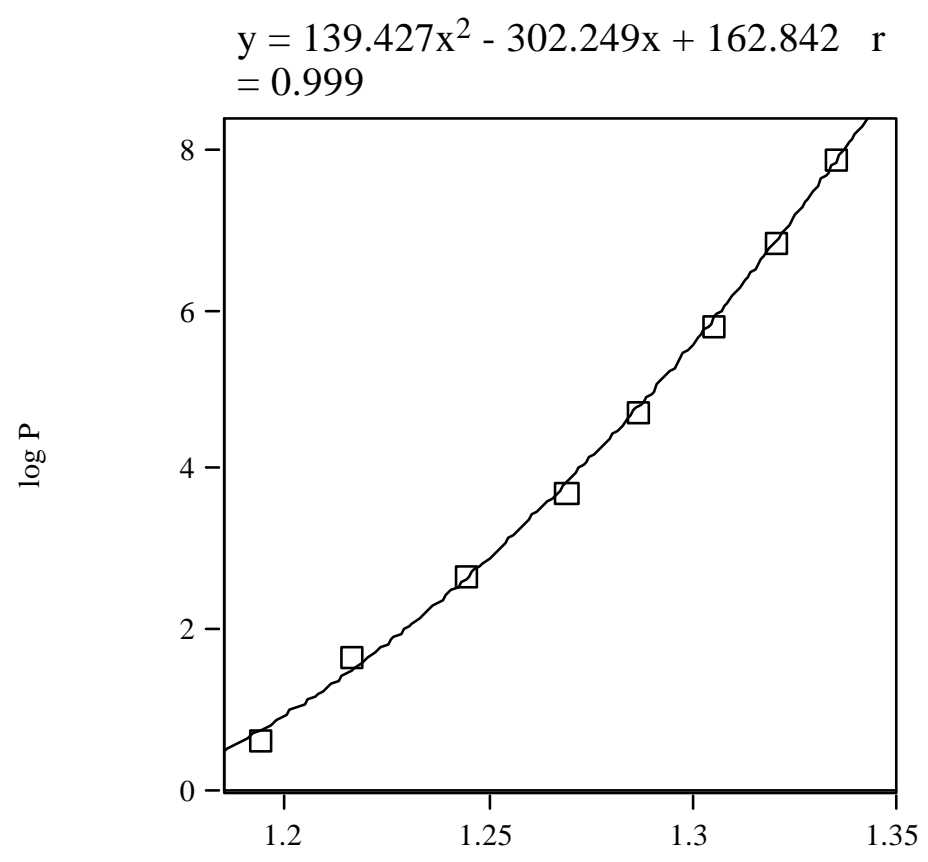

$\mathrm{D}$

The molar concentration necessary to produce a 1:1 complex with bovine serum albumin (BSA) via equilibrium dialysis, $C$, provides information on the binding and conformational perturbation of macromolecules by smaller compounds [56]. It is calculated with SCAP. A non-linear model of $\log 1 / C$ for the homologous phenyl alcohols vs. fractal dimension is illustrated in Figure 4. 
Figure 4. Molar concentration of homologous phenyl alcohols necessary to produce a 1:1 complex with BSA via equilibrium dialysis $v s$. the fractal dimension.

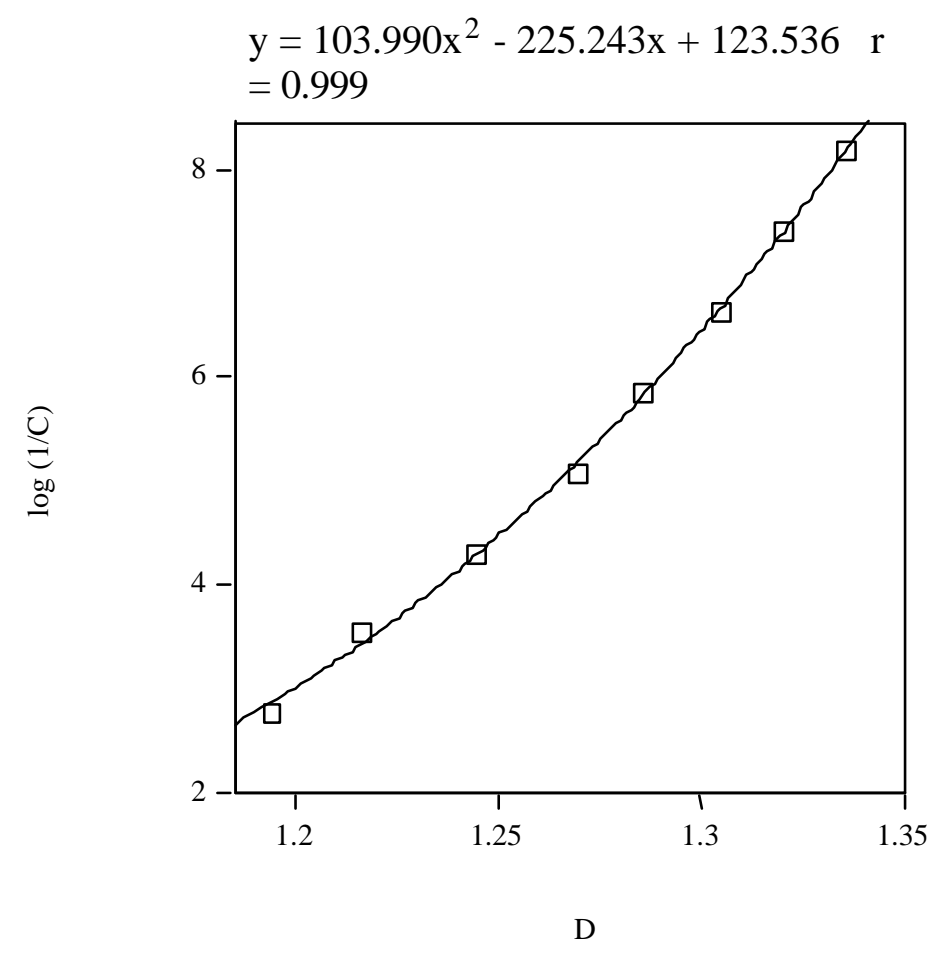

The hydrophile-lipophile balance (HLB) is a scale that characterizes surfactants in emulsions as solubilizers, emulsifiers, wetting agents or antifoaming agents [57,58]. It is calculated with SCAP. A non-linear model of HLB for the homologous phenyl alcohols vs. fractal dimension is shown in Figure 5 .

Figure 5. Hydrophile-lipophile balance of homologous phenyl alcohols vs. the fractal dimension.

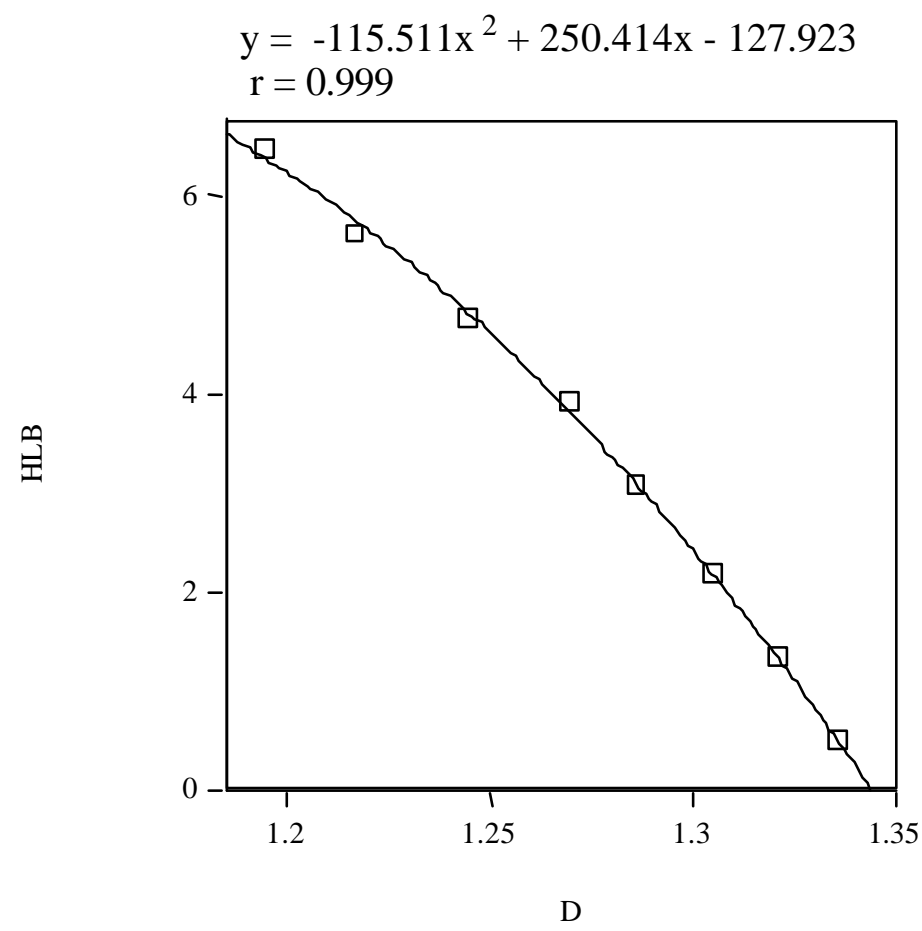


The best linear model for the fractal dimension of the phenyl alcohols $v s$. a series of physicochemical parameters results:

$$
\begin{array}{lrrr}
D=2.51+0.000336 \Theta-0.000140 M_{W}-0.832 G-0.483 G^{\prime} & \text { MAPE }=0.02 \% & \text { AEV }<0.0001 \\
n=8 & r=0.99998 & \mathrm{SD}=0.0006 & F=15570.0
\end{array}
$$

where $M_{W}$ is the molecular weight, $G$ is the molecular globularity and $G^{\prime}$ is the molecular rugosity. Index $G$ is calculated as $G=S_{e} / S$, where $S_{e}$ is the surface area of a sphere whose volume is equal to the molecular volume $V$, and $S$ is the molecular surface area. Index $G^{\prime}$ is calculated as $G^{\prime}=S / V$. All the descriptors are calculated with TOPO. Notice that Equation 15 contains five parameters versus only eight data, allowing three degrees of freedom. The statistical significance of the fit is that the error introduced by fitting is much lower than the error due to the individual points and can be disregarded. Therefore, the data points can be replaced by the fit without adding an appreciable error. The very question is the predictive power of the fit for points out of the fitting set [59]. However, it is not the case because longer phenyl alcohols in the aromatic series are not percutaneous enhancers owing to their lower transdermal penetration. On the other hand, the best non-linear model for the fractal dimension does not improve the results.

\section{Conclusions}

From the preceding results the following conclusions can be drawn:

1. Inclusion of the oxygen atom in the $\pi$-electron system is beneficial for the description of the dipole moment, owing to either the role of the additional $p$ orbitals provided by the heteroatom or the role of steric factors in the $\pi$-electron conjugation. The analysis of both electronic and steric factors in $\mu$ caused by the presence of the oxygen atom shows that the two factors are antagonistic, and that the electronic factor dominates over the steric one.

2. In 3-phenyl-2-propen-1-ol, the double bond in the side chain lends to a more rigid structure with a lower dipole moment.

3. Linear and non-linear correlation models have been obtained for the molecular dipole moments of phenyl alcohols. The new $\mu_{\mathrm{vec}}$ and $\mu_{\mathrm{vec}}^{V}$ charge-transfer indices have improved the multivariable regression equations for $\mu$, diminishing the risk of co-linearity in the fit.

4. The linear correlation between $D$ and $\Theta$, and various non-linear correlations between $D, \log P$, $\log 1 / C$ and HLB point not only to a homogeneous molecular structure of the phenyl alcohols but also to the ability to predict and tailor drug properties. The latter is nontrivial in pharmacology.

Work is in progress on the calculation of the dipole moments of a homologous series of 4-alkylanilines, which are also percutaneous enhancers.

\section{Acknowledgements}

I wish to thank Dr. E. Besalú for providing me several versions of his full linear leave-many-out program prior to publication, and Drs. Gálvez and de Julián-Ortiz for their kind comments. The author 
acknowledges financial support of the Spanish MCT (Plan Nacional I+D+I, Project No. BQU2001-2935-C02-01).

\section{References}

1. Naik, A.; Kalia, Y. N.; Guy, R. H. Transdermal Drug Delivery: Overcoming the Skin's Barrier Function. Pharm. Sci. Technol. Today 2000, 3, 318-326.

2. Langer, R. S. Drug Therapy 1983, 31, 217.

3. Langer, R. S.; Folkman, J. Polymers for the Sustained Release of Proteins and Other Macromolecules. Nature (London) 1976, 263, 797-800.

4. Langer, R. S. Chem. Eng. Commun. 1980, 6, 1.

5. Bunde, A.; Havlin, S.; Nossal, R.; Stanley, H. E.; Weiss, G. H. On Controlled Diffusion-Limited Drug Release from a Leaky Matrix. J. Chem. Phys. 1985, 83, 5909-5913.

6. Kaye, B. H. Fine Particle Characterization Aspects of Predictions Affecting the Efficiency of Microbiological Mining Techniques. Powder Technol. 1987, 50, 177-191.

7. López, A.; Morant, M. J.; Guzmán, D.; Borrás-Blasco, J.; Díez-Sales, O.; Herráez, M. Skin Permeation Model of Phenylalkylcarboxylic Homologous Acids and Their Enhancer Effect on Percutaneous Penetration of 5-Fluorouracil. Int. J. Pharm. 1996, 139, 205-213.

8. López, A.; Pellett, M. A.; Llinares, F.; Díez-Sales, O.; Herráez, M.; Hadgraft, J. The Enhancer Effect of Several Phenyl Alcohols on Percutaneous Penetration of 5-Fluorouracil. Pharm. Res. 1997, 14, 681-685.

9. López, A.; Faus, V.; Díez-Sales, O.; Herráez, M. Skin Permeation Model of Phenyl Alcohols: Comparison of Experimental Conditions. Int. J. Pharm. 1998, 173, 183-191.

10. Yalkowsky, S. H.; Flynn, G. L. Transport of Alkyl Homologues Across Synthetic and Biological Membranes: A New Model for Chain Length-Activity Relationships. J. Pharm. Sci. 1973, 62, 210-216.

11. Flynn, G. L.; Yalkowsky, S. H.; Roseman, T. J. Mass Transport Phenomena and Models: Theoretical Concepts. J. Pharm. Sci. 1974, 63, 479-509.

12. Irwin, W. J.; Sanderson, F. D.; Po, A. L. W. Int. J. Pharm. 1990, 66, 193.

13. Swarbrick, J.; Lee, G.; Brom, J.; Gensmantel, N. P. J. Pharm. Sci., 1984, 73, 1352-1355.

14. Banerjee, P. S.; Ritschel, W. A. Int. J. Pharm. 1989, 49, 189.

15. Lewis, D.; Hadgraft, J. Int. J. Pharm. 1990, 65, 211.

16. Williams, A. C.; Barry, B. W. Pharm. Res. 1991, 8, 17.

17. Saitoh, I.; Ikeda, K.; Takagishi, Y. Effect of Benzyl Alcohol on Rat Skin as a Solvent of Liquid Droplet Dispersion Ointment. Biol. Pharm. Bull. 1995, 18, 321-325.

18. Díez-Sales, O.; Watkinson, A. C.; Herráez-Domínguez, M.; Javaloyes, C.; Hadgraft, J. A Mechanistic Investigation of the in-Vitro Human Skin Permeation Enhancing Effect of Atone ${ }^{\circledR}$. Int. J. Pharm. 1996, 129, 33-40.

19. Rolland, A.; Brzokewicz, A.; Shroot, B.; Jamoulle, J. Int. J. Pharm. 1991, 76, 217. 
20. Torrens, F. A New Topological Index to Elucidate Apolar Hydrocarbons. J. Comput.-Aided Mol. Design 2002, 15, 709-719.

21. Torrens, F. Valence Topological Charge-Transfer Indices for Dipole Moments. J. Mol. Struct. (Theochem), in press.

22. Randi, M. On Characterization of Molecular Branching. J. Am. Chem. Soc. 1975, 97, 6609-6615.

23. Murray, W. J.; Kier, L. B.; Hall, L. H. Molecular Connectivity III: Relationship to Partition Coefficients. J. Pharm. Sci. 1975, 64, 1978-1981.

24. Balaban, A. T. Using Real Numbers as Vertex Invariants for Third-Generation Topological Indexes. J. Chem. Inf. Comput. Sci. 1992, 32, 23-28.

25. Hosoya, H. Topological Index. A Newly Proposed Quantity Characterizing Topological Nature of Structural Isomers of Saturated Hydrocarbons. Bull. Chem. Soc. Jpn. 1971, 44, 2332-2339.

26. Plavsic, D.; Nikolic, S.; Trinajstic, N. On the Harary Index for the Characterization of Chemical Graphs. J. Math. Chem. 1993, 12, 235-250.

27. Gálvez, J.; García, R.; Salabert, M. T.; Soler, R. Charge Indexes. New Topological Descriptors. J. Chem. Inf. Comput. Sci. 1994, 34, 520-525.

28. Gálvez, J.; García-Domenech, R.; de Julián-Ortiz, J. V.; Soler, R. Topological Approach to Drug Design. J. Chem. Inf. Comput. Sci. 1995, 35, 272-284.

29. Gálvez, J.; García-Domenech, R.; de Gregorio-Alapont, C.; de Julián-Ortiz, J. V.; Popa, L. Pharmacological Distribution Diagrams: A Tool for de Novo Drug Design. J. Mol. Graphics 1996, 14, 272-276.

30. Ponce, A. M.; Blanco, S. E.; Molina, A. S.; García-Domenech, R.; Gálvez, J. Study of the Action of Flavonoids on Xanthine-Oxidase by Molecular Topology. J. Chem. Inf. Comput. Sci. 2000, 40, 1039-1045.

31. De Julián-Ortiz, J. V.; García-Domenech, R.; Gálvez-Alvarez, J.; Soler-Roca, R.; García-March, F. J.; Antón-Fos, G. M. Use of Topological Descriptors in Chromatographic Chiral Separations. J. Chromatogr. A 1996, 719, 37-44.

32. De Julián-Ortiz, J. V.; de Gregorio-Alapont, C.; Ríos-Santamarina, I.; García-Domenech, R.; Gálvez, J. Prediction of Properties of Chiral Compounds by Molecular Topology. J. Mol. Graphics Mod. 1998, 16, 14-18.

33. Hocking, R. R. The Analysis and Selection of Variables in Linear Regression. Biometrics 1976, 32, 1-49.

34. Besalú, E. Fast Computation of Cross-Validated Properties in Full Linear Leave-Many-Out Procedures. J. Math. Chem. 2001, 29, 191-203.

35. Buckingham, A. D. Permanent and Induced Molecular Moments and Long-Range Intermolecular Forces. Adv. Chem. Phys. 1967, 12, 107-142.

36. Torrens, F.; Sánchez-Marín, J.; Nebot-Gil, I. Interacting Induced Dipoles Polarization Model for Molecular Polarizabilities. Reference Molecules, Amino Acids and Model Peptides. J. Mol. Struct. (Theochem) 1999, 463, 27-39.

37. Torrens, F. Molecular Polarizability of Fullerenes and Endohedral Metallofullerenes. J. Phys. 
Org. Chem ., 2002, 15, 742-749.

38. Lewis, M.; Rees, D. C. Fractal Surfaces of Proteins. Science 1985, 230, 1163-1165.

39. Torrens, F. Fractal Hybrid Orbitals in Biopolymer Chains. Russ. J. Phys. Chem. (Engl. Transl.) 2000, 74, 115-120.

40. Torrens, F.; Sánchez-Marín, J.; Nebot-Gil, O. New Dimension Indices for the Characterization of the Solvent-Accessible Surface. J. Comput. Chem. 2001, 22, 477-487.

41. Torrens, F. Fractals for Hybrid Orbitals in Protein Models. Complexity Int. 2001, 8, torren01.

42. Torrens, F. Fractal Hybrid Orbitals Analysis of the Tertiary Structure of Protein Molecules. Molecules 2002, 7, 26-37.

43. Torrens, F. Fractal Dimension of Different Structural-Type Zeolites and of the Active Sites. Top. Catal. 2002, 18, 291-297.

44. Torrens, F. Fractal Dimension of Zeolite Catalysts. Mol. Phys. 2002, 100, 3105-3109.

45. Torrens, F. Fractal Dimension of Zeolite Catalysts. Mol. Cryst. Liq. Cryst., submitted for publication.

46. Torrens, F. Fractal Hybrid-Orbital Analysis of the Protein Tertiary Structure. Complexity Int., submitted for publication.

47. Torrens, F. Fractal Dimension of Transdermal-Delivery Drug Models. J. Sci. Libanais, submitted for publication.

48. Torrens, F. Fractal Dimension of Transdermat-Delivery Drug Models: 4-Alkylanilines. Int. J. Quantum Chem ., submitted for publication.

49. Fujita, T.; Iwasa, J.; Hansch, C. A New Substitutent Constant, $\pi$, Derived from Partition Coefficients. J. Am. Chem. Soc. 1964, 86, 5175-5180.

50. Torrens, F.; Sánchez-Marín, J.; Nebot-Gil, I. Universal Model for the Calculation of All Organic Solvent-Water Partition Coefficients. J. Chromatogr. A 1998, 827, 345-358.

51. Torrens, F. Universal Organic Solvent-Water Partition Coefficient Model. J. Chem. Inf. Comput. Sci. 2000, 40, 236-240.

52. Torrens, F. Calculation of Partition Coefficient and Hydrophobic Moment of the Secondary Structure of Lysozyme. J. Chromatogr. A 2001, 908, 215-221.

53. Torrens, F. Free Energy of Solvation and Partition Coefficients in Methanol-Water Binary Mixtures. Chromatographia 2001, 53, S199-S203.

54. Torrens, F.; Soria, V. Stationary-Mobile Phase Distribution Coefficient for Polystyrene Standards. Sep. Sci. Technol. 2002, 37, 1653-1665.

55. Torrens, F. Calculation of Organic Solvent-Water Partition Coefficients of Iron-Sulphur Protein Models. Polyhedron, 2002, 21, 1357-1361.

56. Helmer, F.; Kiehs, K.; Hansch, C. The Linear Free-Energy Relationship Between Partition Coefficients and the Binding and Conformational Perturbation of Macromolecules by Small Organic Compounds. Biochemistry 1968, 7, 2858-2863. 
57. Griffin, W. C. Classification of Surface-Active Agents by "HLB". J. Soc. Cosmet. Chem. 1949, 1, 311-326.

58. Griffin, W. C. Calculation of HLB Values of Non-ionic Surfactants. J. Soc. Cosmet. Chem. 1954, 5, 249-256.

59. Randi, M. The Connectivity Index 25 Years After. J. Mol. Graphics Mod. 2001, 20, 19-35.

60. McClellan, A. L. Tables of Experimental Dipole Moments ; Freeman: San Francisco, 1963.

C 2002 by MDPI (http://www.mdpi.org). Reproduction is permitted for noncommercial purposes 